\title{
Some predictions of the attached eddy model for a high Reynolds number boundary layer
}

\author{
By T. B. Nickels ${ }^{1, *}$, I. Marusic ${ }^{2}$, S. Hafez $^{2}$, \\ N. Hutchins ${ }^{2}$ and M. S. $\mathrm{CHONG}^{2}$ \\ ${ }^{1}$ Department of Engineering, University of Cambridge, \\ Trumpington Street, Cambridge CB2 1PZ, UK \\ ${ }^{2}$ Department of Mechanical and Manufacturing Engineering, \\ University of Melbourne, Victoria 3010, Australia
}

Many flows of practical interest occur at high Reynolds number, at which the flow in most of the boundary layer is turbulent, showing apparently random fluctuations in velocity across a wide range of scales. The range of scales over which these fluctuations occur increases with the Reynolds number and hence high Reynolds number flows are difficult to compute or predict. In this paper, we discuss the structure of these flows and describe a physical model, based on the attached eddy hypothesis, which makes predictions for the statistical properties of these flows and their variation with Reynolds number. The predictions are shown to compare well with the results from recent experiments in a new purpose-built high Reynolds number facility. The model is also shown to provide a clear physical explanation for the trends in the data. The limits of applicability of the model are also discussed.

Keywords: boundary layer; wall turbulence; attached eddy hypothesis; high Reynolds number

\section{The mean velocity profile}

The single most well-known (but not undisputed) classical relationship relating to turbulent wall-bounded flows is the logarithmic dependence of the mean streamwise velocity on the distance normal to the surface. Despite the continuing controversy regarding this 'law', it is still the simplest description of the mean velocity variation that is consistent with experimental data over a wide range of flow conditions. It is discussed here since it is part of the classical description of turbulent boundary layers and its derivation is a good example of the scaling arguments and general approach used later. There are a variety of ways to derive this variation, but here we will appeal to the simplest argument. In general, the mean velocity in a turbulent wall-bounded flow will depend on the wall shear stress, the kinematic viscosity of the fluid, the distance from the wall and the

* Author for correspondence (tbn22@hermes.cam.ac.uk).

One contribution of 14 to a Theme Issue 'Scaling and structure in high Reynolds number wallbounded flows'. 
thickness of the layer. The mean velocity itself, even far from the wall, will also depend on the variation that occurs in the viscous-dominated sublayer and may also be affected by the free-stream velocity. However, it is plausible that the mean velocity gradient in the direction normal to the wall might depend only on local conditions near the point of interest. Using this assumption, we can then imagine a point far from the wall, where viscosity is no longer important, and simultaneously far from the outer edge of the layer so that the outer velocity and length-scale are irrelevant. Hence, the only relevant parameters are the wall shear stress (or equivalently the wall shear velocity) and the distance from the wall. Dimensional analysis then gives

$$
\frac{\mathrm{d} U}{\mathrm{~d} z}=\frac{1}{\kappa} \frac{U_{\tau}}{z},
$$

where $U$ is the mean velocity at some distance $z$ above the wall; $U_{\tau}=\sqrt{\tau_{\mathrm{w}} / \rho}$ is the wall shear velocity; and $\kappa$ is the universal von Karman constant. $\tau_{\mathrm{w}}$ is the shear stress acting on the wall and $\rho$ is the density of the fluid. Equation (1.1) integrates to give the 'log-law', i.e.

$$
U^{+}=\frac{1}{\kappa} \ln \left(z^{+}\right)+C,
$$

where $U^{+}=U / U_{\tau}$ and $z^{+}=z U_{\tau} / \nu$, where $\nu$ is the kinematic viscosity of the fluid. The data presented in $\S 5$ give strong support to this analysis. Note, however, that some researchers dispute the existence of the log-law and offer other alternatives (e.g. Barenblatt et al. 1997; George \& Castillo 1997), but the authors of this paper believe that this classical law has a sound theoretical basis and is well supported by the data. The interested reader is directed to the references cited.

\section{The attached eddy hypothesis}

An attempt will be made here to summarize the main ideas behind the attached eddy hypothesis of Townsend (1976) and the extension of that hypothesis to the model of Perry \& Chong (1982). The aim is to lay down the essential features of the structure of turbulent boundary layers according to the model and point out those that may be tested using the experimental results available.

In a book remarkable for its sheer density of original ideas, Townsend (1976) laid out a model for the structure of wall-bounded equilibrium layers based on arrays of large 'eddies'. This was, in part, inspired by the success of similar large eddies in explaining the correlation measurements of Grant (1958) in a plane wake. These eddies may be thought of as the velocity fields of some representative vortex structures.

Given the apparent success of the log-law in describing the mean velocity profile, with characteristic insight Townsend notes, 'it is difficult to imagine how the presence of the wall could impose a dissipation length-scale proportional to distance from it unless the main eddies of the flow have diameters proportional to distance of their 'centres' from the wall, because their motion is directly influenced by its presence. In other words, the velocity fields of the main eddies, regarded as persistent, organized flow patterns, extend to the wall and, in a sense, they are attached to the wall'. To put it more simply, any eddy with a size 
that scales with its distance from the wall may be considered to be attached to the wall. Eddies further from the wall are larger in size and hence their velocity fields still extend to the wall (of course the velocity fields of vortex structures extend to infinity but they decay rapidly at large distances).

It is eddies of this type that form the basis of the attached eddy hypothesis. The hypothesis itself is that the main energy-containing motion of a turbulent wall-bounded flow may be described by a random superposition of such eddies of different sizes, but with similar velocity distributions. These eddies should be considered as statistically representative structures in that their geometry and strength are derived from an ensemble average of many different structures of similar scale. As such there may be no single eddy in the flow with this precise structure (further explanation of this concept may be found in Nickels \& Marusic 2001).

Townsend then formed an expression for the contribution of a random superposition of attached eddies of different sizes to the correlation functions and, using the zero-penetration boundary condition at the wall, derived the distribution of eddy sizes with wall distance necessary to produce the observed invariance of the Reynolds shear stress $\left(\overline{u w} / U_{\tau}^{2}\right)$ with distance from the wall. This analysis effectively leads to a population density of eddies that varies inversely with size and hence with distance from the wall. Simply, the number of eddies of size $z$ per unit wall area is $A / z$ where $A$ is a constant. Using this, population density also leads to predictions for the variation of the other (normal) components of the Reynolds stress, i.e.

$$
\frac{\overline{u^{2}}}{U_{\tau}^{2}}=C_{1}+D_{1} \log \left(\frac{\delta}{z}\right), \quad \frac{\overline{v^{2}}}{U_{\tau}^{2}}=C_{2}+D_{2} \log \left(\frac{\delta}{z}\right) \quad \text { and } \quad \frac{\overline{w^{2}}}{U_{\tau}^{2}}=C_{3},
$$

where $\overline{u^{2}}, \overline{v^{2}}$ and $\overline{w^{2}}$ are the streamwise, spanwise and wall-normal components of the Reynolds stress, respectively, $\delta$ is the boundary layer thickness and $C_{1}, C_{2}$, $C_{3}, D_{1}$ and $D_{2}$ are constants. It may be noted that so far very little has been specified about the eddies and the behaviour essentially arises due to the zeropenetration boundary condition at the wall and the invariance of $-\overline{u w} / U_{\tau}^{2}$. The choice of the detailed structure of the eddies changes the values of the constants.

The physical understanding of these relationships rests essentially on the fact that the normal to the wall component and the shear stress component at a given height, $z$, are mainly due to the influence of eddies with 'centres' at or close to $z$, whereas the streamwise and spanwise fluctuations at a given distance from the wall are due to all eddies with heights greater than $z$. That is why these components increase as the distance from the wall is reduced: as we approach the wall the number of eddies larger than our distance from the wall increases (see Perry et al. 1986). Naturally, there is a limit to this increase since viscosity will set a limit on the size of the smallest possible attached eddy. In the model of Perry \& Chong (1982), this limit is approximately 100 wall units $\left(100 \nu / U_{\tau}\right)$, although its exact value is not critical.

The motions due to the larger eddies (well above the point of interest) are mainly 'sweeping' or 'sloshing' motions which carry very little shear stress since the scale of the flows is so large that they appear to be mostly parallel to the wall on the scale of the local distance from the wall. It is these flows that Townsend called 'inactive' since they carry little shear stress. It is important to note, 
however, that as Townsend explains '.. the inactive flow at one level is an essential part of active flow at other higher levels...' In other words, when measuring at a particular distance from the wall, $z$, the active motions come from eddies with centres located at or near $z$, whereas the inactive motions are due to eddies much larger than $z$. Which motions are considered active and inactive depends on the location of the measurement.

The expressions above really only apply to the 'main turbulent motion', which essentially relates to that region where the important length-scale is distance from the wall and the important velocity scale is the wall shear velocity, in other words the log-law region. Since the size of the eddies scales with $z$ and their velocities with $U_{\tau}$, this is nicely consistent with the scaling arguments used to derive the log-law.

In order to proceed further, Townsend had to specify the detailed structure of the eddies. He felt that conical eddies were appropriate and gave fairly good agreement with available correlation measurements. He did note, however, that 'Other possibilities exist, e.g. a distribution of shorter double-roller eddies of various sizes each with a lateral extent comparable with distance from the wall...'

Perry \& Chong (1982) developed a model for the mechanism of wall turbulence in which the flow is made up of a superposition of attached eddies. Rather than conical eddies, they had vortex loops in mind, in which the vortex starts near or on the wall, rises above the wall and then bends back to meet the wall again. These have variously been called hairpin eddies, horse-shoe eddies and lambda eddies and are consistent with the flow visualization studies of Head \& Bandyopadhyay (1981). In the first part of their paper, they concentrated on using such eddies to model the mean flow. They recognized that the gradient of the mean velocity in the wall-normal direction is due to the mean cross-stream (spanwise) vorticity component of the superposition of attached eddies. Taking a random distribution of such eddies with a population density that varies inversely with size (as in Townsend's attached eddy model), they found that the logarithmic velocity profile could be reproduced. As a result of the similarity with the attached eddy model of Townsend, they also found the same behaviour for the Reynolds stresses as in equation (2.1). In addition, they considered the resulting spectral distribution of energy for the model and found that, again primarily due to the population density, this results in a $k_{1}^{-1}$ power law spectrum for the streamwise velocity fluctuation (where $k_{1}$ is the streamwise wavenumber) in a region close enough to the wall. Perry \& Chong (1982) also made the connection between the spectra and the stresses and pointed out that a $k_{1}^{-1}$ spectrum integrates to give a logarithmic dependence of the streamwise stress on the wall-normal position as given in equation (2.1). Note that a precise $k^{-1}$ region in the spectrum is not necessary for the streamwise stress to exhibit a logarithmic dependence since the integral is not sensitive to small departures from this behaviour. It is also worth noting here that the particular conical eddies favoured by Townsend would not produce a $k_{1}^{-1}$ law in the streamwise component and this is reflected in the equations for the stresses since the conical eddies give $D_{1}=0$ in equation (2.1).

Physically, in terms of the attached eddy model, the occurrence of a $k_{1}^{-1}$ region in the spectrum can be explained in terms of the velocity signatures of attached eddies. The argument follows the same reasoning as given above for the stresses. At a given distance from the wall, $z$, a probe measuring streamwise or spanwise fluctuations will only register contributions from eddies of size greater than or 
equal to $z$. The largest eddy contributing to the $k_{1}^{-1}$ region may be expected to be of size $\approx 0.1 \delta$ (since this is the largest eddy within the logarithmic region of the flow). The smallest eddy that contributes is of order of the distance from the wall, $z$. If we then wish to estimate the length of the $k_{1}^{-1}$ region, we find the ratio of contributing scales is $\approx 0.1 \delta / z$. In order to see a decade of $k_{1}^{-1}$ in the spectrum, we would need $0.1 \delta / z \approx 10$ and the distance from the wall would need to be $z / \delta<0.01$. It turns out that this estimate is a little generous and the measurements presented later show that the distance needs to be even less (Nickels et al. 2005 estimated that $z / \delta<0.002$ is required for one decade). This explains why Morrison et al. (2004) and McKeon \& Morrison (2007) did not observe any $k_{1}^{-1}$ region in their spectrum since the lowest level examined was $z / \delta=0.03$ where a $k_{1}^{-1}$ region in the spectrum cannot be expected. At this point, the ratio of the largest to the smallest attached eddy in the turbulent wall region and contributing to the streamwise stress is only three at the most.

A further point should be noted. While it is necessary to approach the wall closely in order to observe $k_{1}^{-1}$ behaviour, the closeness of approach is limited by the effects of viscosity which become more important nearer the wall. Results, to be presented later, suggest that these effects are quite small at $z^{+} \geq 100$. McKeon \& Morrison (2007) have examined this question in terms of the local Reynolds number required to establish a Kolmogorov-type inertial range in the spectrum. The idea is that a sufficient scale separation is required between the large eddies in the flow and those responsible for the dissipation. This can be related to the value of the Taylor microscale Reynolds number, $R_{\lambda}$. McKeon \& Morrison (2007) consider previous research and available data and suggest a value of $R_{\lambda} \geq 100$ as a sufficient criterion.

This very brief summary of the attached eddy model and the developments of it by Perry \& Chong (1982) and others is sufficient to provide a basis for understanding the predictions of this 'classical' approach for high Reynolds number boundary layers. Note that the approach here is considered classical in that it fits nicely within the classical framework for high Reynolds number boundary layers in terms of the appropriate scaling arguments and underlying structure. The physics follows logically from the derivation of the logarithmic velocity variation for the boundary layer and it makes testable predictions for the spectra and stresses. It should be noted here that this model has extensively been refined and developed in the last 20 years and the reader is referred to Perry \& Marusic (1995) and Marusic (2001) for a summary of some recent developments and further details of the model.

One refinement examined in Marusic (2001) was the incorporation of long structures into the basic model. These structures lead to a low-wavenumber 'hump' in the streamwise spectrum which is not present in predictions from the attached eddy model. Marusic (2001) found that this could be accounted for by considering 'packets' of attached eddies, aligned in the streamwise direction, as the basic structural unit of the flow. In the original model of Perry \& Chong (1982), individual eddies were considered uncorrelated and hence the longest extent of streamwise correlation could only be of the size of the largest eddy in the flow (of order $\delta$, the boundary layer thickness). This simple modification leaves the general structure and predictions of the model unchanged but replicates the contributions of these longer structures very well. Hence, the presence of long structures is compatible with the attached eddy model. The size 
of this hump in the energy spectrum seems to increase with Reynolds numbers for low Reynolds number flows (see Kim \& Adrian 1999; Hutchins \& Marusic 2007); however, indications are that this increase either stops or becomes quite small beyond $\delta^{+} \approx 10000$. This may be confirmed by examining fig. 4 in Nickels et al. (2005) in which two Reynolds numbers are shown and, perhaps more convincingly by comparing these results to those presented in McKeon \& Morrison (2007) in the Princeton superpipe. The Reynolds number differs by a factor of two between these results (in different flows) and yet the height of the low wavenumber hump is virtually the same (this does not, of course, preclude a very slow growth in the height of the hump). A further comparison can be made with other superpipe data presented in Morrison et al. (2004) in which the Reynolds number is higher by a factor of 10 . Here there appears to be a small increase in the maximum height of the peak of approximately $15 \%$. A final comparison may be made with the atmospheric data of Kunkel \& Marusic (2006) in the atmospheric boundary layer. While these measurements (in common with virtually all atmospheric data) suffer from lack of complete convergence at the low wavenumbers, and hence have a very large scatter, it is still possible to make a rough estimate of the height of the hump. These measurements with a Reynolds number approximately 400 times as high as in Nickels et al. (2005) give a best estimate of hump height only approximately $20 \%$ greater. It appears then that if there is any increase at higher Reynolds numbers it is very small. Particular attention has been paid to this issue since it has been suggested that the growth of this peak might 'swamp' the occurrence of a $k_{1}^{-1}$ region in the spectra. This now seems unlikely at all terrestrial Reynolds numbers.

\section{Measurements of boundary layers at high Reynolds number}

In order to examine any predictions regarding the structure of turbulent boundary layers at high Reynolds number, it is first necessary to make careful and accurate measurements of such flows. These measurements are not trivial and can suffer from uncertainties due to the establishment of the flow and the resolution of the measurement technique. In this section, we discuss some of the issues relating to the resolution of the measurements before going on to examine the actual measurements that have been made to test the predictions of the models discussed above.

Hot-wire anemometry is one of the most commonly used measuring techniques for turbulence research. It involves measuring the heat transfer from a very small heated wire and hence inferring the velocity of the fluid passing over the wire. While the sensors used in turbulence research are very small (typically from 0.2 to $1 \mathrm{~mm}$ in length and 1 to $5 \mu \mathrm{m}$ in diameter), they still have finite dimensions and can only resolve motions in the flow larger than the wire length. Smaller motions are spatially averaged over the length of the wire. Since the scales of motion in turbulence at high Reynolds number can become very small, this spatial averaging can lead to misleading results if it is not considered carefully. Eddies of smaller size than the wire length will not fully contribute to the higher order statistics of the flow. The inability to resolve these small eddies is relevant for turbulence measurements of flows at high Reynolds number and for measurements close to solid boundaries. This is a direct result of the reduction in the size of the small scale viscous dissipative eddies and also a reduction in the size of the smallest 
anisotropic ('attached') eddy as the Reynolds number is increased. Ligrani \& Bradshaw (1987) carried out extensive normal hot-wire measurements in a boundary layer flow with $R_{\theta}=2620$. Near-wall measurements with subminiature normal hot-wire sensors revealed that a wire of length, $l^{+} \equiv l U_{\tau} / \nu<20-25$ is sufficient to yield turbulence intensity measurements within $10 \%$ of the 'true' values all the way to the wall. They also suggested that wires with an aspect ratio (length/diameter) of approximately 200 will produce a uniform temperature along the wire length that will reduce the end conduction effects and improve the temporal resolution of the wire. These limits are commonly used as a guide for selecting sensors for the turbulence measurements. The effect of the sensor size in turbulence measurements has also been investigated by Hites (1997) in a high Reynolds number boundary layer. Normal hot-wire measurements were carried out along a cylindrical model with a diameter of $45.7 \mathrm{~cm}$ and length of $900 \mathrm{~cm}$. Different wires with diameters ranging from 0.5 to $3.8 \mu \mathrm{m}$ were tested at two measuring stations. The first measuring station was located at $184 \mathrm{~cm}$ from the tripping device. At this station $R_{\theta}$ varied from 4100 to 9720 , corresponding to viscous length, $\nu / U_{\tau}$, of 26 to $11 \mu \mathrm{m}$. The second measuring station was $733 \mathrm{~cm}$ from the tripping device with free-stream velocity of $28.6 \mathrm{~m} \mathrm{~s}^{-1}, R_{\theta}=19300$ and $\nu / U_{\tau}=17 \mu \mathrm{m}$. Turbulence intensity profiles measured with two different wires, $l^{+}=6$ and 31, which should not collapse due to the large difference in $l^{+}$, showed complete collapse. This implies that a wire length of $l^{+}=30$ is sufficiently small to resolve these turbulent flows. This is inconsistent with the findings of Ligrani \& Bradshaw (1987). Given the inconsistency in the literature, the safest approach for an experimentalist is to test for spatial resolution effects in the flow of interest by using sensors of different sizes.

\section{Apparatus and experimental techniques}

The test facility was purpose built for the study of high Reynolds number, turbulent boundary layers and consists of an open return blower wind tunnel with a $27 \mathrm{~m}$ working length and a $2 \times 1 \mathrm{~m}$ cross-section. The flow conditioning consists of a honeycomb section, a perforated plate followed by six screens and a contraction of area ratio 6.25 , where the aspect ratio is held constant along the length to minimize corner flow influences (Callan \& Marusic 2001). The maximum speed of the wind tunnel is $45 \mathrm{~m} \mathrm{~s}^{-1}$ and it has a free-stream turbulence intensity of $0.05 \%$ (at $30 \mathrm{~m} \mathrm{~s}^{-1}$ ). Measurements were carried out for boundary layers developing on the tunnel floor, which is covered by aluminium plates of $6 \times 2 \mathrm{~m}$ in size and $6 \mathrm{~mm}$ in thickness. The surface roughness of these plates is $1.5 \mu \mathrm{m}$ (r.m.s.) as measured with a Perthometer M3. Measurements for the main study, developing flows, were limited to three reference unit Reynolds numbers $U_{\infty} / \nu=6.48 \times 10^{5}, 1.33 \times 10^{6}$ and $1.94 \times 10^{6}$ per metre. They correspond to nominal reference free-stream velocity, $U_{\infty}$, of 10,20 and $30 \mathrm{~m} \mathrm{~s}^{-1}$, respectively (some measurements were also taken at $40 \mathrm{~m} \mathrm{~s}^{-1}$ for one downstream station). Further measurements at matched Reynolds number, constant $R_{\theta}$, were carried out at three reference unit Reynolds numbers of $6.48 \times 10^{5}, 1.03 \times 10^{6}$ and $1.59 \times 10^{6}$ per metre, and the corresponding nominal reference free-stream velocity of 10, 16 and $24 \mathrm{~m} \mathrm{~s}^{-1}$, respectively. Ambient flow conditions were measured using a calibrated thermocouple and an electronic barometer. 
A nominally zero pressure-gradient was maintained along the working section using a series of $1 \mathrm{~m}$ width adjustable ceiling panels. Air bleeding and adjusting the height of these panels were the two mechanisms used to control the streamwise pressure variation. A biplane screen, $25 \mathrm{~mm}$ square-mesh with $3 \mathrm{~mm}$ wire diameter, was placed at the exit of the working section so as to increase the pressure in the working section and facilitate the bleeding of air. The adjustable ceiling panels were located sufficiently far from the plate such that the flow near the boundary layer on the floor was as for a normal zero pressuregradient boundary layer. A similar technique was used by Marusic \& Perry (1995) to impose various pressure gradients on a boundary layer. Careful measurements of their flow showed that the bleeding did not have any detrimental effect on the normal development of the layer. It was possible to maintain the pressure-coefficient $\left(C_{\mathrm{p}}\right)$ variation along the entire measuring section of the tunnel, to within $\pm 0.7 \%$ for the 20 and $30 \mathrm{~m} \mathrm{~s}^{-1}$ cases, but for the $10 \mathrm{~m} \mathrm{~s}^{-1}$ case, the $C_{\mathrm{p}}$ distribution varied by $\pm 1.0 \%$. Mean flow velocity profiles were measured using $1.0 \mathrm{~mm}$ total head Pitot tube and with single normal hot-wires. The static pressure was measured by a static tube placed approximately $25 \mathrm{~mm}$ above the total head tube. The Pitot tube readings were corrected for the effect of shear using the MacMillan (1956) correction. The Clauser chart technique was used employing traditional constants, $\kappa=0.41$ and $A=5.0$, to deduce local skin friction coefficient, $C_{\mathrm{f}}=2 \tau_{\mathrm{w}} / \rho U_{1}^{2}$. After conducting a careful study of tripping devices and locations, the decision was made to trip all boundary layers developing along the inner surface of the contraction using sandpaper sheets, grade 40 of $115 \mathrm{~mm}$ width, placed $750 \mathrm{~mm}$ upstream from the exit.

The normal single sensor probe is a DANTEC 55P05 and was used with a constant temperature anemometer system (AN-1003 from AA lab systems) operating at an overheat ratio of 1.8. The frequency response of the system to a $2 \mathrm{kHz}$ internal pulse was greater than $200 \mathrm{kHz}$. Wollaston wires are soldered to the probe and etched to give a platinum filament with core diameters of 5.0 and $2.5 \mu \mathrm{m}$, with active lengths of approximately 0.9 and $0.4-0.6 \mathrm{~mm}$, respectively. A static calibration technique, with a third-order polynomial curve fit, is used to convert the measured anemometer output voltage into velocity. The normal hot-wire is statically calibrated against a Pitot-static tube pair. The uncertainty in the wall distance is estimated to be $\pm 5 \mu \mathrm{m}$. Hot-wire signals were sampled online using a Microstar 16 bit data acquisition board model DAP3000a/21. Turbulence intensity measurements were taken in bursts of 8000 samples and four bursts were found to be sufficient to obtain results converged to within $1 \%$. The signals were sampled at $200 \mathrm{~Hz}$ and filtered at $20 \mathrm{kHz}$. The $u$-spectra were measured with calibrated normal wires and the signals were sampled at $80 \mathrm{kHz}$ and low-pass filtered at $32 \mathrm{kHz}$. Data were sampled in bursts of $2^{18}$ points and 500 bursts were found to give converged results. More details regarding spectra measurements can be found in Nickels et al. (2005). Taylor's hypothesis of frozen turbulence was used to transform the spectral argument from the frequency domain, $f$, to the wavenumber domain $k_{1}$, such that

$$
k_{1}=\frac{2 \pi f}{U_{\mathrm{c}}},
$$




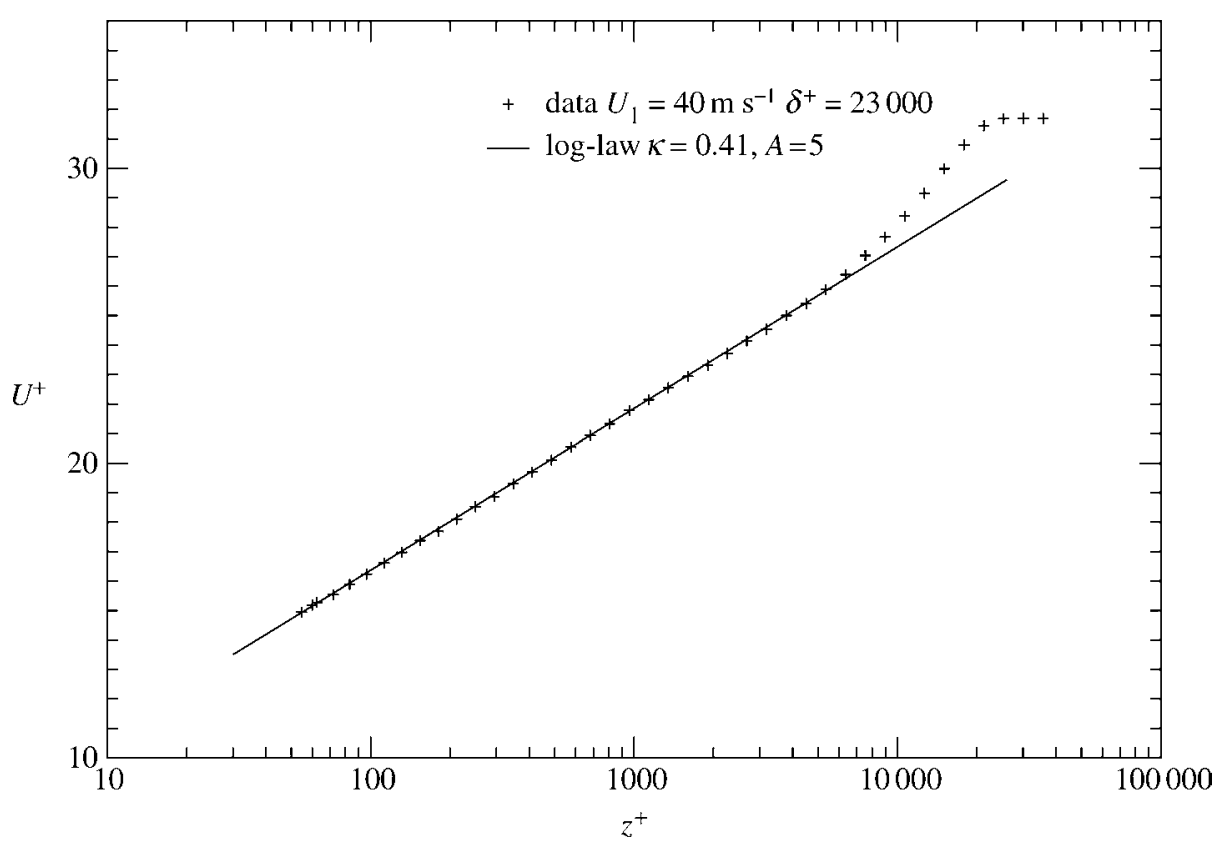

Figure 1. Log-law for highest Reynolds number $\left(R_{\theta}=64000\right)$ mean velocity profile. The symbol size indicates the uncertainty in the measurements.

where $U_{\mathrm{c}}$ is the local mean convection velocity, which is assumed to be equal to the local mean velocity. The spectra were normalized such that

$$
\int_{0}^{\infty} \phi_{11}\left(k_{1}\right) \mathrm{d} k_{1}=\overline{u^{2}}
$$

where $\phi_{11}\left(k_{1}\right)$ is the power spectral density (PSD) per unit streamwise wavenumber $k_{1}$.

\section{Results and discussion}

\section{(a) Mean flow results}

In order to demonstrate the applicability and extent of the logarithmic law derived earlier, figure 1 shows the log-law compared with the variation of the mean velocity for the highest Reynolds number boundary layer measured. The log-law for these data certainly seems to be a good approximation over two decades in wall distance where it lies well within the (small) uncertainty of the data. Further measurements were made at eight streamwise stations with the first station located $0.4 \mathrm{~m}$ after the tripping device and the last station at $21.7 \mathrm{~m}$. Detailed mean flow analysis can be found in Hafez et al. (2004). Mean flow velocity profiles for the developing flow are shown in inner flow scaling in figure 2. The profiles are also compared with the log-law of the wall employing traditional constants. Apart from a slight overshoot within the buffer region, there is very good collapse of the data in the turbulent wall region, TWR, across the full Reynolds number range, $1.1 \times 10^{3}<R_{\theta}<5.2 \times 10^{4}$. Here, the TWR is defined 


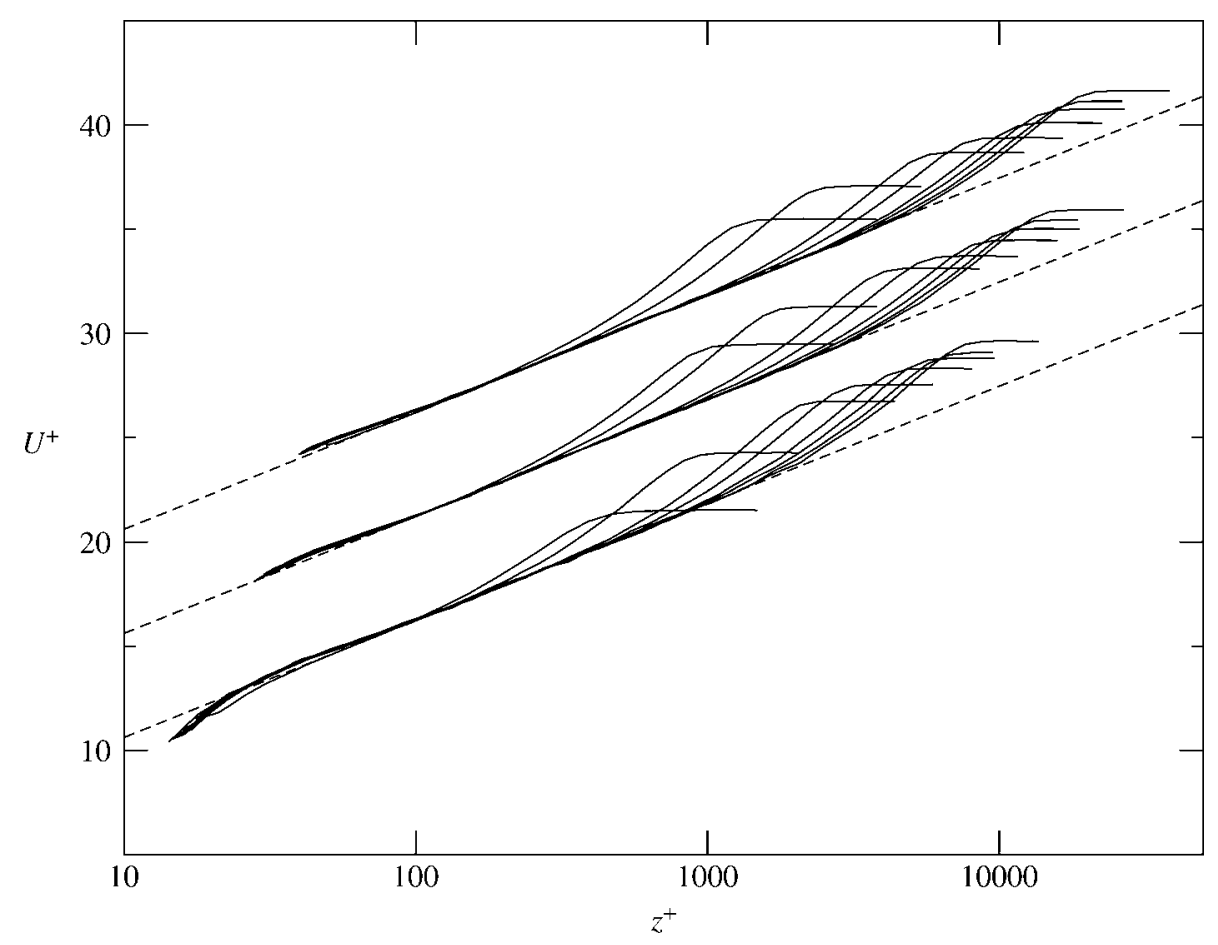

Figure 2. Mean low-velocity profiles with inner flow scaling. Profiles are shifted up 5 units for the $20 \mathrm{~m} \mathrm{~s}^{-1}, 10$ units for the $30 \mathrm{~m} \mathrm{~s}^{-1}$ and no change for the $10 \mathrm{~m} \mathrm{~s}^{-1}$; dashed-line represents log-law, $\kappa=0.41$ and $A=5.0$. Reynolds number range: $1.1 \times 10^{3}<R_{\theta}<5.2 \times 10^{4}$.

as $z^{+}>100$ and $z / \delta<0.15$. Outside the TWR, deviation from the log-law and later blending with the background irrotational flow occurs smoothly. It should be noted that the collapse of the data with inner scaling is due to the use of the Clauser-chart method for estimating $U_{\tau}$. The logarithmic variation of the mean flow, however, is unaffected by the method of analysis.

\section{(b) Streamwise stress}

\section{(i) Spatial resolution}

Figure 3 shows the streamwise turbulence intensity profiles taken for positions with the same Reynolds number $\left(R_{\theta} \approx 18000\right)$, but with different wire lengths. We might expect the attenuation to extend to a wall distance which is some multiple of $l^{+}$and an examination of the results suggests that the attenuation starts to occur where $z^{+}<3 l^{+}$, approximately. The $10 \mathrm{~m} \mathrm{~s}^{-1}$ case represents the most resolved measurement in our study $\left(l^{+} \approx 10\right.$ and 22 shown in the graph) and the only small differences between the two different wire lengths are limited to values of $z^{+}<60$. This result is in agreement with the finding of Ligrani \& Bradshaw (1987), but not with Hites (1997). The data of Hites at $R_{\theta}=19000$ (as discussed earlier), were measured with wires length $l^{+}$of 6 and 31. Hites' data gave a peak value of approximately 7.3 at about $z^{+}=15$, and roughly constant value of 5.8 for $70<z^{+}<700$. These values agree well with the present 


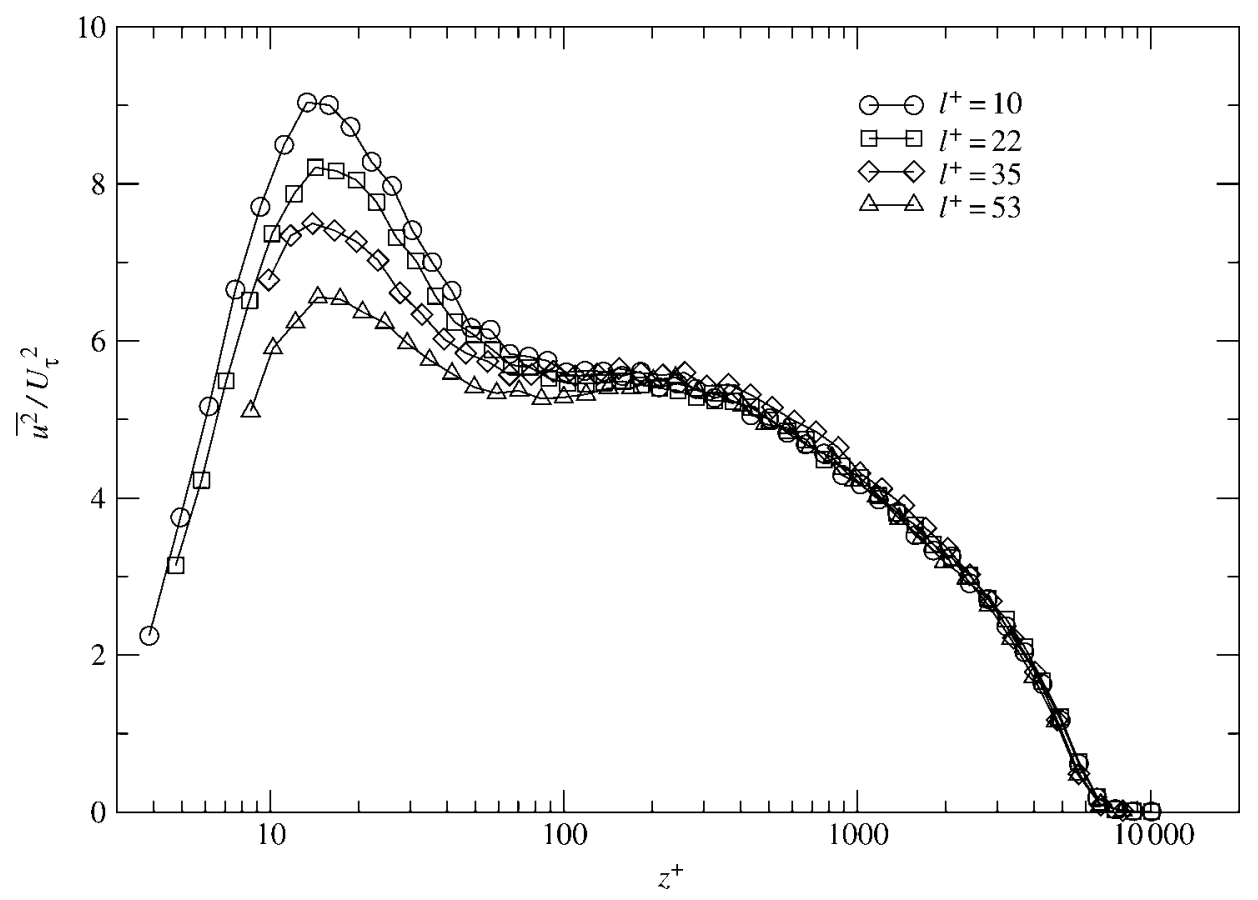

Figure 3. Effect of spatial resolution. Streamwise turbulence intensity profiles at the same Reynolds number $\left(R_{\theta} \approx 18000\right)$, but with different non-dimensional wire lengths.

measurements at $24 \mathrm{~m} \mathrm{~s}^{-1}$ and wire length $l^{+}$of 32.4 , which is attenuated due to spatial resolution. It is odd that the $l^{+}=6$ measurements appear to be attenuated despite the nominally good spatial resolution. Another interesting feature of the plots is that the highly attenuated profiles, $l^{+}=35$ and 53 , can be seen to have double peaks, one close to the wall and the other further away (the peak for $l^{+}=35$ is harder to see on this plot due to the number of profiles shown). This behaviour represents an attenuation of turbulence energy over a large portion of the boundary layer. Similar trends have been observed in boundary layer flows by Fernholz et al. (1995) and more recently by Morrison et al. (2004) in which a peak is becomes apparent when $l^{+}>30$ : this is also when it becomes apparent in the results shown above. This observation is consistent with an explanation for its appearance as being due to insufficient spatial resolution. An interesting feature of this second, spurious, outer peak in our results is that its position depends on $l^{+}$. In the case of $l^{+}=35$, it is at $z_{\mathrm{p}}^{+}=170$ and for $l^{+}=53$, it is at $z_{\mathrm{p}}^{+}=240$. Since $z_{\mathrm{p}}^{+}$varies with $l^{+}$then if a wire of a fixed physical length is used to measure a flow with changing Reynolds number (which leads to changing $l^{+}$) then the position of this peak will appear to be a function of Reynolds number. This is an example of the way in which insufficient spatial resolution can give misleading results. In the case of all of the other results presented here, the worst spatial resolution of the hot-wire was $l^{+}=32$ and conclusions are only based on measurements taken in regions of the flow where the attenuation due to spatial resolution was negligible. Inadequate spatial resolution is, then, one possible explanation for the appearance and movement of 


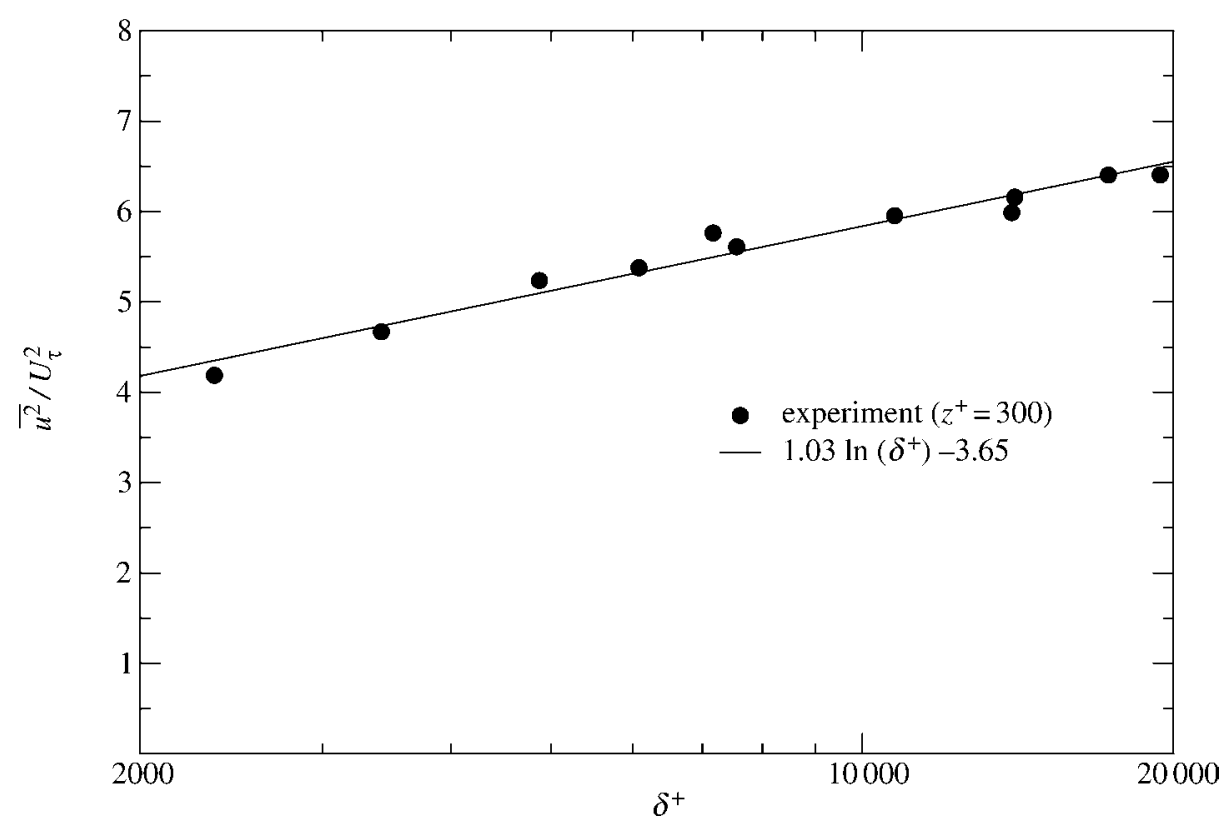

Figure 4. Variation of the streamwise turbulence intensity at $z^{+}=300$ versus $\delta^{+}$. Data compared with the attached eddy prediction.

the outer peak in the streamwise Reynolds stress observed by Fernholz et al. (1995) and Morrison et al. (2004). Measurements with much smaller probes are planned for the superpipe at Princeton and should help to resolve this issue.

\section{(ii) Predictions of the attached eddy model}

Since we are interested in predicting the behaviour of boundary layers at high Reynolds number, it is useful to examine the prediction of the attached eddy model for the variation of the streamwise stress with Reynolds number. Equation (2.1) can be rewritten

$$
\frac{\overline{u^{2}}}{U_{\tau}^{2}}=C_{1}+D_{1} \log \left(\frac{\delta}{z}\right)=C_{1}+D_{1} \log \delta^{+}-D_{1} \log z^{+} .
$$

If we now examine a fixed value of $z^{+}=300$, say, then we have an equation for the variation of the streamwise stress at this non-dimensional location with the Reynolds number, $\delta^{+}$. Figure 4 shows the result compared with the theory and the agreement is quite good over about a decade in Reynolds number. There are two reasons for choosing the value of $z^{+}=300$. The first is that this is far enough from the wall for the effects of spatial resolution to be negligible for all the measurements and the second is that Marusic et al. (1997) have shown that there is a correction to the basic behaviour which varies approximately as $\left(z^{+}\right)^{-1 / 2}$ which is fairly small at $z^{+}=300$. There are actually several corrections which account for viscous effects and outer flow effects, but these have been neglected here since they are not significant in this case. These corrections should be seen as refinements to the essential attached eddy model and approach. The reader is 
directed to the reference for further information. The value of $D_{1}$ used here should be universal and is the same as that used in Marusic et al. (1997) to analyse other data $\left(D_{1}=1.03\right)$. The model is particularly useful since it not only gives a good prediction of the functional form of the relation but also, more importantly, gives a simple physical explanation for the increase with Reynolds number. Fixing $z^{+}$and increasing the Reynolds number, $\delta^{+}$means moving closer to the wall since $z / \delta=z^{+} / \delta^{+}$. As noted earlier, moving closer to the wall leads to an increase in the streamwise (and spanwise) stress due to the increased number of eddies that are above the probe position and hence contribute to these quantities. Kunkel \& Marusic (2006) also showed that the logarithmic dependence is apparent in the atmospheric boundary layer at $\delta^{+} \approx 4 \times 10^{6}$ - two orders of magnitude higher than the measurements shown here (with the same constant, $D_{1}$ ). The model has also recently been extended by Marusic \& Kunkel (2003) to predict the near-wall peak of the streamwise stress that occurs in the buffer region of the layer $\left(\right.$ at $\left.z^{+} \approx 15\right)$.

\section{(iii) Spectra}

As mentioned earlier, the attached eddy model as extended by Perry \& Chong (1982) also predicts a $k_{1}^{-1}$ variation for the streamwise spectra. This variation can also be derived from scaling arguments, but implicit in these arguments is a structure consistent with the attached eddy model discussed here. Essentially, it assumes that there is a range of sizes of eddies in the flow with characteristic velocities that scale with $U_{\tau}$ and with sizes that scale with $z$. From this basis there are various ways of deriving the $k_{1}^{-1}$ behaviour, for example an overlap argument as used by Nickels et al. (2005). Essentially, the idea is that there is a range in wavenumber space in which the effects of both viscosity and the outer length-scale (e.g. the boundary layer thickness or the pipe radius) are negligible. The essential feature of this region is that an overlap exists where both inner and outer scaling are simultaneously valid. Inner scaling here refers to non-dimensionalization using the length-scale $z$, the wall-normal position and the velocity scale $U_{\tau}$, the friction velocity. Outer scaling uses the length-scale $\delta$, the boundary layer thickness and the same velocity scale $U_{\tau}$. Inner scaling requires that

$$
\frac{\phi_{11}\left(k_{1} z\right)}{U_{\tau}^{2}}=\frac{\phi_{11}\left(k_{1}\right)}{z U_{\tau}^{2}}=f\left(k_{1} z\right)
$$

and outer flow scaling requires that

$$
\frac{\phi_{11}\left(k_{1} \delta\right)}{U_{\tau}^{2}}=\frac{\phi_{11}\left(k_{1}\right)}{\delta U_{\tau}^{2}}=f\left(k_{1} \delta\right),
$$

where $\phi_{11}\left(k_{1} z\right)$ is the PSD of the streamwise velocity fluctuation per unit nondimensional wavenumber $k_{1} z$. If there is a region where these two scalings are simultaneously valid, then the streamwise spectrum (PSD) must vary as $k_{1}^{-1}$. Figure 5 shows spectra measured in the Melbourne facility taken from Nickels et al. (2005). The Reynolds number for this flow is $\delta^{+} \approx 15000$, which is comparable with the Reynolds numbers examined in McKeon \& Morrison (2007; figure 4) from the Princeton superpipe. In this plot, the spectra have been premultiplied by $k_{1}$ such that a plateau on the plot corresponds to the $k_{1}^{-1}$ 


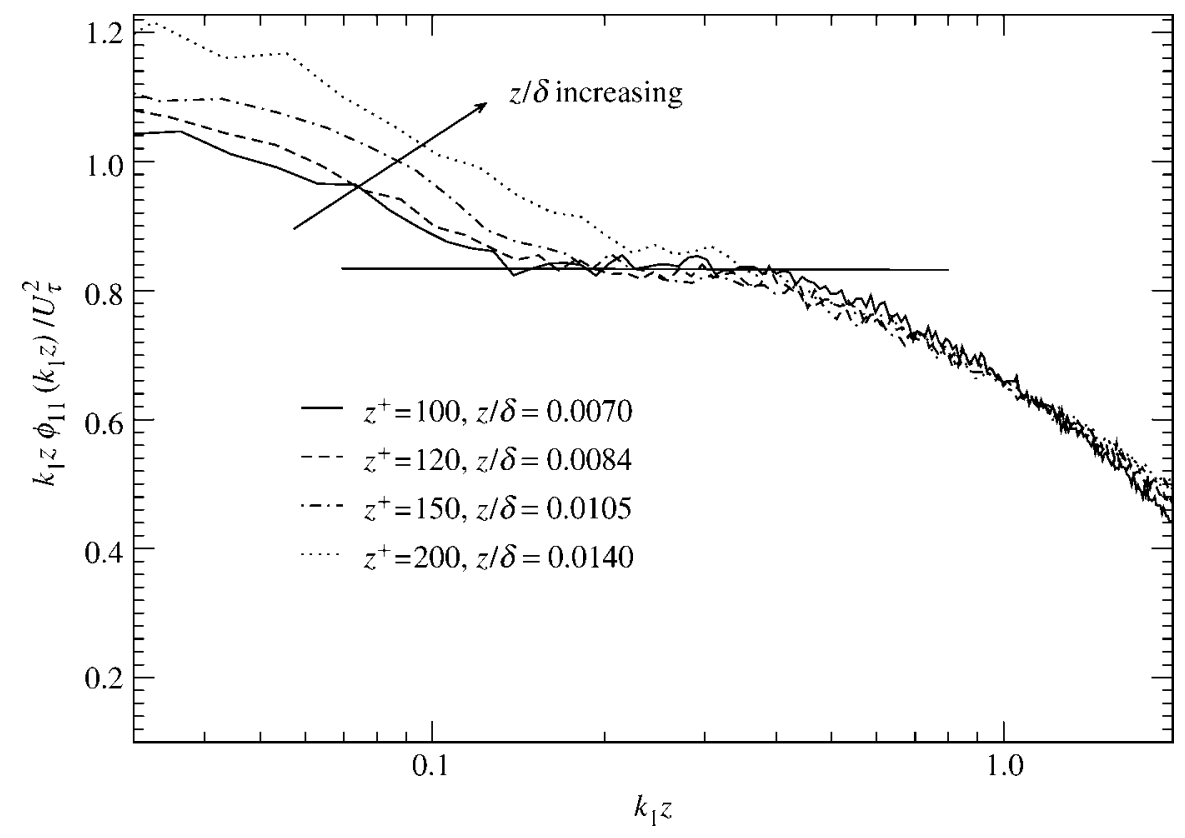

Figure 5. Spectra showing $k^{-1}$ region.

behaviour. On this plot, which is scaled with the distance from the wall $z$, the curves peel-off from the plateau as the wall-distance is increased - a behaviour which is consistent both with the scaling arguments and the attached eddy model. When plotted scaled with outer scaling, the profiles collapse at the low wavenumber end and peel-off at the high end - consistent with the scaling arguments and the attached eddy model. The reader is directed to the reference for more details regarding both the measurements and the conclusions drawn from these measurements. For the purposes of this paper, it is sufficient to show that there is a region of $k^{-1}$ behaviour in the streamwise spectra and its appearance, location and scaling are all consistent with the attached eddy model as discussed here. An important point should be stressed here. The authors have chosen to present the results premultiplied and in close up to show the variation of the levels. Even the results that peel-off are quite close to $k^{-1}$ behaviour if the numbers are compared. When this plot is shown in non-premultiplied form in the usual way (on $\log -\log$ axes), the $k^{-1}$ slope appears to cover almost two decades. That is why the integration of the spectra still effectively gives logarithmic behaviour for the stresses even where the close-up premultiplied spectrum appear to have peeled-off from the $k^{-1}$ plateau. The level shown that is closest to the wall is at $z^{+}=100$. As mentioned earlier, it is important that the effects of viscosity should be small at this level. Following the analysis of McKeon \& Morrison (2007), it is possible to work out the value of $R_{\lambda}$ at this level. The value is $R_{\lambda}=147$ which comfortably exceeds the minimum requirement of $R_{\lambda} \geq 100$ as suggested in their paper.

It is also worth pointing out that the model predicts the same behaviour for the spectrum of the spanwise velocity component, $\phi_{22}\left(k_{1}\right)$, but different behaviour for the spectrum of the wall-normal component, $\phi_{33}\left(k_{1}\right)$. Using the argument that the contributions to the wall-normal component come mostly 
from eddies of size $z$, we then expect inner scaling for $\phi_{33}\left(k_{1}\right)$, but not outer scaling and hence there is no overlap. Kunkel \& Marusic (2006) have shown that inner scaling does indeed collapse $\phi_{33}\left(k_{1}\right)$, over a range of three orders of magnitude in Reynolds number, whereas outer scaling does not work at all and, as a result, there is no $k^{-1}$ region apparent in the spectrum. This observation provides additional support for the model.

\section{Conclusions}

It has been shown that the attached eddy model of the turbulent boundary layer provides testable predictions of the behaviour of these flows at high Reynolds numbers. It does this by postulating a particular underlying physical structure for the flow and then developing the implications of such a structure for the measurable statistical properties of the flow such as the mean velocity, the Reynolds stresses and the spectra. While some of this behaviour might be predicted based on other assumptions, the beauty of the model is that it ties together scaling arguments, mean-flow behaviour, Reynolds stresses and spectra in one internally consistent physical model. The empirical evidence presented here is consistent with the predictions of the model and lends support to the underlying ideas. The experiments presented for comparison are unique in providing wellconverged data measured with good spatial resolution at high Reynolds number. High Reynolds number data of this quality are necessary in order to test the predictions of this, and other, models. The quality of the measurements presented here has been achieved through the careful design and construction of the purposebuilt high Reynolds number facility at the University of Melbourne.

S.H., N.H. and M.S.C. wish to acknowledge the financial assistance of an Australian Research Council (ARC) Discovery grant. I.M. would like to acknowledge support from the NSF and the Packard Foundation. T.B.N. is grateful for the support received from EPSRC(GR/S86303/01). The authors also gratefully acknowledge financial contributions towards the construction of the experimental facility from ARC-Discovery and ARC-Facility grants.

\section{References}

Barenblatt, G. I., Chorin, A. J., Hald, O. H. \& Prostokishin, V. M. 1997 Structure of the zeropressure-gradient turbulent boundary layer. Proc. Natl Acad. Sci. USA 94, 7187-7819.

Callan, J. \& Marusic, I. 2001 Effects of changing aspect ratio through a wind tunnel contraction. AIAA J. 39, 1800-1803.

Fernholz, H. H., Krause, K., Nockemann, M. \& Schober, M. 1995 Comparative measurements in the cannonical boundary layer at $R e_{\delta 2} \leq 6 \times 10^{4}$ on the wall of the German-Dutch windtunnel. Phys. Fluids 7, 1275-1281. (doi:10.1063/1.868516)

George, W. K. \& Castillo, L. 1997 Zero-pressure gradient turbulent boundary layer. Appl. Mech. Rev. 50, 689-729.

Grant, H. L. 1958 The large eddies of turbulent motion. J. Fluid Mech. 4, 149-190. (doi:10.1017/ S0022112058000379)

Hafez, S., Jones, M. B. \& Chong, M. S. 2004 The zero pressure gradient turbulent boundary layer and its approach to equilibrium. In Proc. 10th Asian Fluid Mech. Conf., Peradeniya, Sri Lanka.

Head, M. R. \& Bandyopadhyay, P. 1981 New aspects of turbulent boundary layer structure. J. Fluid Mech. 107, 297-338. (doi:10.1017/S0022112081001791) 
Hites, M. H. 1997 Scaling of high-Reynolds number turbulent boundary layers in the National Diagnostic Facility. Ph.D. thesis, Illinois Institute of Technology.

Hutchins, N. \& Marusic, I. 2007 Large-scale influences in near-wall turbulence. Phil. Trans. R. Soc. A 365, 647-664. (doi:10.1098/rsta.2006.1942)

Kim, K. C. \& Adrian, R. J. 1999 Very large-scale motion in the outer layer. Phys. Fluids 11, 417-422. (doi:10.1063/1.869889)

Kunkel, G. J. \& Marusic, I. 2006 Study of the near-wall-turbulent region of the high-Reynoldsnumber boundary layer using an atmospheric flow. J. Fluid Mech. 548, 375-401. (doi:10.1017/ S0022112005007780)

Ligrani, P. M. \& Bradshaw, P. 1987 Spatial resolution and measurement of turbulence in the viscous sublayer using subminiature hot-wire probes. Exp. Fluids 5, 407-417. (doi:10.1007/ BF00264405)

MacMillan, F. A. 1956 Experiments on Pitot-tubes in shear flow. Reports and memoranda No. 3028, Aeronaut. Res. Council.

Marusic, I. 2001 On the role of large-scale structures in wall turbulence. Phys. Fluids 13, 735-743. (doi:10.1063/1.1343480)

Marusic, I. \& Kunkel, G. J. 2003 Streamwise turbulence intensity formulation for flat-plate boundary layers. Phys. Fluids 15, 2461-2464. (doi:10.1063/1.1589014)

Marusic, I. \& Perry, A. E. 1995 A wall-wake model for the turbulence structure of boundary layers. Part 2. Further experimental support. J. Fluid Mech. 298, 389-407. (doi:10.1017/ S0022112095003363)

Marusic, I., Uddin, A. K. M. \& Perry, A. E. 1997 Similarity law for the streamwise turbulence intensity in zero-pressure-gradient turbulent boundary layers. Phys. Fluids 9, 3718-3724. (doi:10.1063/1.869509)

McKeon, B. J. \& Morrison, J. F. 2007 Asymptotic structure and inertial scaling in wall-bounded flow. Phil. Trans. R. Soc. A 365, 771-787. (doi:10.1098/rsta.2006.1945)

Morrison, J. F., McKeon, B. J., Jiang, W. \& Smits, A. J. 2004 Scaling of the streamwise velocity component in turbulent pipe flow. J. Fluid Mech. 508, 99-131. (doi:10.1017/S0022112004008985)

Nickels, T. B. \& Marusic, I. 2001 On the different contributions of coherent structures to the spectra of a turbulent round jet and a turbulent boundary layer. J. Fluid Mech. 448, 367-385. (doi:10.1017/S002211200100619X)

Nickels, T. B., Marusic, I., Hafez, S. \& Chong, M. S. 2005 Evidence for the $k^{-1}$ law in a high Reynolds number boundary layer. Phys. Rev. Lett. 95, 074501-1-074501-4. (doi:10.1103/ PhysRevLett.95.074501)

Perry, A. E. \& Chong, M. S. 1982 On the mechanism of wall turbulence. J. Fluid Mech. 119, 173-217. (doi:10.1017/S0022112082001311)

Perry, A. E. \& Marusic, I. 1995 A wall-wake model for the turbulence structure of boundary layers. Part 1. Extension of the attached eddy hypothesis. J. Fluid Mech. 298, 361-388. (doi:10.1017/ S0022112095003351)

Perry, A. E., Henbest, S. \& Chong, M. S. 1986 A theoretical and experimental study of wall turbulence. J. Fluid Mech. 165, 163-199. (doi:10.1017/S002211208600304X)

Townsend, A. A. 1976 The structure of turbulent shear flow, 2nd edn. Cambridge, UK: Cambridge University Press. 


\section{University Library}

\section{- M M I N E R VA A gateway to Melbourne's research publications}

Minerva Access is the Institutional Repository of The University of Melbourne

\section{Author/s:}

Nickels, T. B.;Marusic, I.;Hafez, S.;Hutchins, N.;Chong, M. S.

Title:

Some predictions of the attached eddy model for a high Reynolds number boundary layer

Date:

2007-01

Citation:

Nickels, T. B., Marusic, I., Hafez, S., Hutchins, N., \& Chong, M. S. (2007). Some predictions of the attached eddy model for a high Reynolds number boundary layer. Philosophical Transactions of the Royal Society A, 365(1852), 807-822.

Publication Status:

Published

Persistent Link:

http://hdl.handle.net/11343/34800 